\title{
Sensor Drift Compensation Using Fuzzy Interference System and Sparse-Grid Quadrature Filter in Blood Glucose Control ${ }^{\star}$
}

\author{
Péter Szalay ${ }^{1}$, László Szilágyi ${ }^{1}$, Zoltán Benyó ${ }^{1}$, and Levente Kovács ${ }^{2}$ \\ 1 Department of Control Engineering and Information Technology, \\ Budapest University of Technology and Economics, Budapest, Hungary \\ \{szalaip, Iszilagyi, benyo\}@iit.bme.hu \\ 2 Applied Informatics Institute, John von Neumann Faculty of Informatics, \\ Obuda University, Budapest, Hungary \\ kovacs.levente@nik.uni-obuda.hu
}

\begin{abstract}
Diabetes mellitus is a serious chronic condition of the human metabolism. The development of an automated treatment has reached clinical phase in the last few years. The goal is to keep the blood glucose concentration within a certain region with minimal interaction required by the patient or medical personnel. However, there are still several practical problems to solve. One of these would be that the available sensors have significant noise and drift. The latter is rather difficult to manage, because the deviating signal can cause the controller to drive the glucose concentration out of the safe region even in the case of frequent calibration. In this study a linear-quadratic-Gaussian (LQG) controller is employed on a widely used diabetes model and enhanced with an advanced Sparse-grid quadratic filter and a fuzzy interference system-based calibration supervisor.
\end{abstract}

Keywords: Diabetes, LQG control, Sparse-grid quadratic filter, fuzzy inference system.

\section{Introduction}

The blood glucose concentration is regulated through a complex endocrine system of the human body, where insulin plays a key role. If the glucose-insulin interaction is impaired, diabetes is diagnosed. Artificial Pancreas (AP) is a mean to provide an automated treatment of the insulin dependent type-1 diabetes (T1DM) by keeping the blood glucose levels of the patient in normoglycemic range $(3.9-7.8 \mathrm{mmol} / \mathrm{L})$. It consists of three main parts: a continuous glucose monitor (CGM), an insulin pump for subcutaneous delivery and a control algorithm for closed-loop control.

\footnotetext{
* Research supported by the Hungarian National Research Funds (OTKA), Project no. PD103921 and by the European Union TÁMOP-4.2.2.A-11/1/KONV-2012-0073 project. Levente Kovács is Bolyai Fellow of the Hungarian Academy of Sciences.
} 
The aim of the AP is to automatically regulate the blood glucose concentration of T1DM patients with minimal interaction required, ensuring reasonable safety in all times. There are various methods to choose from: classical PID [1; run-to-run control 2]; exact linearization based nonlinear control 3]; $\mathcal{H}_{\infty}$ control [45]; Model Predictive Control (MPC) 617]; Linear Parameter Varying (LPV) control 8 819, among others. Soft computing based methods, such as fuzzy logic control [10] and model-free soft computing-based control [11] are gaining popularity as well. Most of these techniques require signals beyond what is physically measurable; hence, accurate estimation of the state variables is needed.

However, most commercially available CGMs do not measure the glucose concentration directly, but the time derivative instead, therefore the readings will slowly drift from the actual values. Hence, calibration is needed. In this work it will be investigated how nonlinear stochastic filtering and fuzzy interferencebased timing of the calibration can ensure satisfactory operation of the AP.

\section{Diabetes Model}

The employed model is described by the following differential equations [12]:

$$
\begin{aligned}
\dot{C}(t)= & -k_{a, i n t} C(t)+\frac{k_{a, i n t}}{V_{G}} Q_{1}(t) \\
\dot{Q}_{1}(t)= & -\left(\frac{F_{01}}{Q_{1}(t)+V_{G}}+x_{1}(t)\right) Q_{1}(t)+k_{12} Q_{2}(t)- \\
& -R_{c l} \max \left\{0, Q_{1}(t)-R_{t h r} V_{G}\right\}-P h y(t)+ \\
& +E G P_{0} \max \left\{0,1-\frac{S_{I E} k_{b 1} k_{a}}{V_{I} k_{e}} S_{2}(t)\right\}+\min \left\{U_{G, c e i l}, \frac{G_{2}(t)}{t_{\text {max }}}\right\} \\
\dot{Q}_{2}(t)= & x_{1}(t) Q_{1}(t)-\left(k_{12}+\frac{S_{I D} k_{b 1} k_{a}}{V_{I} k_{e}} S_{2}(t)\right) Q_{2}(t) \\
\dot{x}_{1}(t)= & -k_{b 1} x_{1}(t)+\frac{S_{I T} k_{b 1} k_{a}}{V_{I} k_{e}} S_{2}(t) \\
\dot{S}_{2}(t)= & -k_{a} S_{2}(t)+k_{a} S_{1}(t) \\
\dot{S}_{1}(t)= & -k_{a} S_{1}(t)+u(t) \\
\dot{G}_{2}(t)= & \left(G_{1}(t)-G_{2}(t)\right) / \max \left\{t_{\text {max }}, G_{2}(t) / U_{G, c e i l}\right\} \\
\dot{G}_{1}(t)= & -G_{1}(t) / \max \left\{t_{\text {max }}, G_{2}(t) / U_{G, c e i l}\right\}+D(t)
\end{aligned}
$$

where the state variables are: $C(t)$ glucose concentration in the subcutaneous tissue $[\mathrm{mmol} / \mathrm{L}] ; Q_{1}(t), Q_{2}(t)$ the masses of glucose in accessible and nonaccessible compartments [mmol]; $x_{1}(t)$ remote effect of insulin on glucose distribution [1/min]; $S_{1}(t), S_{2}(t)$ insulin masses in the accessible and non-accessible compartments [mU]; $G_{1}(t), G_{2}(t)$ glucose masses in the accessible and nonaccessible compartments [mmol]. $u(t)$ injected insulin flow of rapid-acting insulin 
$[\mathrm{mU} / \mathrm{min}]$ is the input of the system, while $D(t)$ amount of ingested carbohydrates $[\mathrm{mmol} / \mathrm{min}]$, and $P h y(t)$ effect of physical activity $[\mathrm{mmol} / \mathrm{min}]$ represent the disturbances 12. All parameters are assumed to be time-invariant.

Based on [12] and [13] the model is reduced to a 9th order one (three neglected states) as the linear transfers of the reduced states have time constants comparable to the sampling time $\left(T_{s}\right)$ of the used sensor. As CGM measurements are available every 5 minutes, the following discrete-time sensors model is used:

$$
x_{d}[k+1]=x_{d}[k]+w_{d}[k] \quad y_{1}[k]=x_{d}[k]+C[k]+z_{1}[k]
$$

where $C[k]=C\left(k \cdot T_{s}\right), x_{d}[k]$ is the state variable associated with sensor drift, $y_{1}[k]$ is the output of the sensor, finally $w_{d}[k]$ and $z_{1}[k]$ are white noises with Gaussian distribution. The former represents the disturbance that drives the sensor drift with $0.1667 \mathrm{mmol}^{2} / L^{2}$ variance, while the latter is additive measurement noise with $0.25 \mathrm{mmol}^{2} / L^{2}$ variance. Because of the drift, the sensor must be re-calibrated using a manual glucose measurement $y_{2}[k]=C[k]+z_{2}[k]$, which is assumed to have only additive measurement noise $z_{2}[k]$ with very small variance $0.0025 \mathrm{mmol}^{2} / \mathrm{L}^{2}$ compared to sensor noise. However, these manual measurements cannot be done very often.

\section{Closed-Loop Control}

Here a relatively simple control method is proposed as the main focus of this paper is not on the control algorithm: Linear-quadratic-Gaussian (LQG) control [14]. It is basically a moving horizon model predictive control, which minimizes a quadratic constraint for the state variables and control signal, which is as follows:

$$
J(\mathrm{x}, \mathrm{u})=\frac{1}{2} \sum_{k=0}^{N-1}\left(<\mathbf{Q}_{k} \mathrm{x}[k], \mathrm{x}[k]>+<\mathbf{R}_{k} \mathrm{u}[k], \mathrm{u}[k]>\right)+\frac{1}{2}<\mathbf{Q}_{N} \mathrm{x}[N], \mathrm{x}[N]>
$$

where $J(\mathrm{x}, \mathrm{u})$ is defined for an $N$ sample-long time horizon, $\mathrm{x}[k]$ represents the vector of state variables and $u[k]$ the controlled input. $\langle$.$\rangle denotes the scalar$ product, while $\mathbf{Q}_{k}$ and $\mathbf{R}_{k}$ are appropriately chosen symmetric and positive definite matrices. In the case of a linear time-invariant system given in statespace form with matrices $\mathbf{A}, \mathbf{B}$ and $\mathbf{C}$ and time-invariant weighting matrices $\mathbf{Q}$ and $\mathbf{R}$ as well as infinite time horizon $(N=\infty), J(\mathrm{x}, \mathrm{u})$ can be minimized using state-feedback control $\mathrm{u}[k]=\mathbf{K x}[k]$. The gain for the feedback can be acquired by solving Discrete-time algebraic Riccati equation:

$$
\begin{aligned}
& \mathbf{X}=\mathbf{Q}+\mathbf{A}^{T} \mathbf{X A}-\mathbf{A}^{T} \mathbf{X B}\left(\mathbf{B}^{T} \mathbf{X B R}\right)^{-1} \mathbf{B}^{T} \mathbf{X A} \\
& \mathbf{K}=\left(\mathbf{B}^{T} \mathbf{X B R}\right)^{-1} \mathbf{B}^{T} \mathbf{X A}
\end{aligned}
$$

Since model (1) is nonlinear, it is approximated with a linear model in every step $k$, then the gain for the state feedback $\mathrm{K}[k]$ is computed. For better disturbance rejection integral control has been included [14] and the linear model extended accordingly. The control law is as follows:

$$
\begin{aligned}
& x_{i}[k+1]=x_{i}[k]+y[k]-4.9 \\
& u[k]=\mathrm{K}[k]\left(\left(\mathrm{x}_{b} 0\right)^{T}-\left(\mathrm{x}[k] x_{i}[k]\right)^{T}\right)+u_{b}
\end{aligned}
$$


where $\mathrm{x}_{b}$ is the steady state value of the state variables, $x_{i}[k]$ is the state variable corresponding to integral control, and $u_{b}$ is the steady state control input for zero meal intake and $4.9 \mathrm{mmol} / \mathrm{L}$ normoglycemic blood glucose concentration denoted with $y[k]=Q_{1}[k] / V_{G}$. For $n \times n$ matrix $\mathbf{Q}$ and matrix $\mathbf{R}$ (that is a scalar) used in the cost function (3) the following values were selected:

$$
\mathbf{Q}=\mathrm{e}_{1} \mathrm{e}_{1}^{T}+\mathrm{e}_{n} \mathrm{e}_{n}^{T} \quad \mathbf{R}=10
$$

where $\mathrm{e}_{i}$ denotes the $i$-th unit vector in $\mathbb{R}^{n}$. The controller was tuned for extreme meal intake and physical activity scenario detailed later in Section 6.

\section{Observer Design}

Since both significant disturbances, measurement noise and sensor drift are present state estimation is required using Kalman filtering technique. Sigmapoint filters are an increasingly popular option in the case of nonlinear models [15. These use a special set of points - called sigma points - to estimate the mean and covariance of distributions needed in the Kalman filter algorithm. A good example would be the Gauss-Hermite Quadrature Filter (GHQF) [16. It offers high accuracy when all disturbances and measurement noises have Gaussian distribution, but requires a large number of sigma-points. Hence, it needs relatively large computational power undesirable in certain practical applications. Sparsegrid quadrature nonlinear filtering (SGQF) can overcome this dimensionality problem [15, and consequently was chosen for our application as well.

\subsection{Sigma-Point Selection}

Let us introduce the notation $\chi$ for a set of sigma-points. This set contains $N$ sigma points denoted as $\xi_{i}, i=1, \ldots, N$. The sigma-points represent the stochastic variable $\mu$ with mean $\hat{\mu}$ and covariance matrix $\boldsymbol{\Sigma}$, and can be written in the form: $\xi_{i}=\boldsymbol{\Sigma}^{\frac{1}{2}} \varphi_{i}+\hat{\mu}$. $\Sigma^{\frac{1}{2}}$ is the factor of $\boldsymbol{\Sigma}$ so that $\boldsymbol{\Sigma}=\boldsymbol{\Sigma}^{\frac{1}{2}} \boldsymbol{\Sigma}^{\frac{T}{2}}$, and since $\boldsymbol{\Sigma}$ is positive definite the Cholesky decomposition is used. $\varphi$ is an additional vector used for sigma point determination [15. $\mu$ is not limited to state variables only, it can contain the disturbances and measurement noises as well. The dimension of $\mu$ will be denoted with $L$. GHQF requires $m^{L}$ sigma points, where $m$ is usually set to 3. Level-3 SGQF provides a good approximation of the results of GHQF, but requires only $2 L^{2}+4 L+1$ or less sigma-points. The exact number depends on how the three parameters of sigma-point filter $\left(p_{1}, p_{2}, p_{3}\right)$ are set [15. They reflect the case of univariate estimation, where the points $\mu+\left\{-p_{1}, 0, p_{1}\right\}$ and $\mu+\left\{-p_{3},-p_{2}, 0, p_{2}, p_{3}\right\}$ are used to estimate certain moments of a univariate Gaussian distribution transformed by a nonlinear function. If all parameters are different the sigma-points used in the level-3 SGQF are shown in equations (77) and (8), where $C=L(L-1) / 2$, while $\hat{\omega}_{1}, \ldots, \hat{\omega}_{5}$ are defined from the parameters $p_{1}, \ldots, p_{3}$ using moment matching method. 


$$
\begin{aligned}
& \omega_{i}=\left\{\begin{array}{cc}
\frac{(L-1)\left(L-2+L \hat{\omega}_{1}^{2}\right)}{2}-L(L-1) \hat{\omega}_{1}+L \hat{\omega}_{3} & i=1 \\
(L-1) \hat{\omega}_{2}\left(\hat{\omega}_{1}-1\right) & i=2, \ldots, 2 L+1 \\
\hat{\omega}_{4} & i=2 L+2, \ldots, 4 L+1 \\
\hat{\omega}_{5} & i=4 L+2, \ldots, 6 L+1 \\
\hat{\omega}_{2}^{2} & i=6 L+2, \ldots, 6 L+1+4 C
\end{array}\right. \\
& \varphi_{i}=\left\{\begin{array}{cc}
0 & i=1 \\
\mathrm{e}_{i} p_{1} & i=2, \ldots, L+1 \\
-\mathrm{e}_{i} p_{1} & i=L+2, \ldots, 2 L+1 \\
\mathrm{e}_{i} p_{2} & i=3 L+2, \ldots, 4 L+1 \\
-\mathrm{e}_{i} p_{2} & i=4 L+2, \ldots, 5 L+1 \\
\mathrm{e}_{i} p_{3} & i=5 L+2, \ldots, 6 L+1 \\
-\mathrm{e}_{i} p_{3} & i=6 L+2, \ldots, 6 L+1+C j \neq i \\
\mathrm{e}_{i} p_{1}+\mathrm{e}_{j} p_{1}, & i=6 L+2+C, \ldots, 6 L+1+2 C j \neq i \\
-\mathrm{e}_{i} p_{1}+\mathrm{e}_{j} p_{1}, & i=6 L+2, \ldots, 6 L+1+3 C j \neq i \\
\mathrm{e}_{i} p_{1}-\mathrm{e}_{j} p_{1}, & i=6 L+2+2 C, \ldots, 6 L+1+4 C j \neq i \\
-\mathrm{e}_{i} p_{1}-\mathrm{e}_{j} p_{1}, i=6 L+2+3 C, \ldots, 6 L+1+4
\end{array}\right.
\end{aligned}
$$

The complete discrete-time nonlinear system created from the T1DM model (11) using Euler method, and the sensor model (2) is of the following form:

$$
\mathrm{x}[k+1]=\mathrm{f}(\mathrm{x}[k])+\mathbf{B} \mathrm{w}[k] \quad \mathrm{y}[k]=\mathbf{C x}[k]+\mathrm{z}[k]
$$

where $\mathrm{x}[k]=\left(C[k], Q_{1}[k], Q_{2}[k], x_{1}[k], S_{2}[k], S_{1}[k], G_{2}[k], G_{1}[k], x_{d}[k]\right)^{T}$ and $\mathrm{y}[k]=\left(y_{1}[k], y_{2}[k]\right)^{T}$ or only $y_{1}[k]$ depending on whether manual measurement is available or not.

\section{$5 \quad$ Fuzzy Calibration Supervisor}

Since the CGM has drift, it needs to be repeatedly re-calibrated. The used filter can compensate to some degree; however, the state estimation will eventually deviate from the true value, and the controller relying on those estimations will malfunction as well. The goal is to keep the glucose concentration levels within normoglycemic range, and most importantly to avoid hypoglycemia. However, the manual measurements needed for calibration cannot be made too frequently. Furthermore, the patient must sleep, and hence calibration is only allowed at night if it is to avoid a potentially life-threatening situation. Mamdani Fuzzy Interference System was used to determine the timing of the calibration. There are six inputs:

1. Time passed since the last manual measurement. The fuzzy sets associated with this input represent that the last calibration was: not long ago $(<60-90$ min), really long ago (> 12 hours), or neither; 
2. Actual time. The fuzzy sets associated are that the patient is probably sleeping (from around $11 \mathrm{pm}$ to around $6 \mathrm{am}$ ), or that the patient is about to go to sleep;

3. $7.8-\hat{C}[k]-\sqrt{\left[\Sigma_{x x}\right]_{1,1}[k]}$, where $\hat{C}[k]$ is the estimated glucose concentration in the subcutaneous tissue, $\left[\Sigma_{x x}\right]_{1,1}[k]$ is the variance of the estimation error $C[k]-\hat{C}[k] .7 .8 \mathrm{mmol} / L$ is the lower bound of severe hyperglycemia. There are three fuzzy sets: one associated with severe hyperglycemia, one representing when the signal might be close to the border, finally one capturing the case when the signal is far even considering the estimation error;

4. The time derivative of the above signal; The decreasing and increasing trend of this signal is captured by two fuzzy sets.

5. $\hat{C}[k]-\sqrt{\left[\Sigma_{x x}\right]_{1,1}[k]}-3.9$, since the glucose concentration decreasing below $3.9 \mathrm{mmol} / \mathrm{L}$ is considered hypoglycemia. The fuzzy sets are the same as for the third input;

6. The time derivative of the above signal. Same fuzzy sets as in the case of the 4th input;

The rules used are as follows:

- If a lot of time has passed since the last calibration, a manual measurement is needed, however

- If the last measurement was only a short time ago, there must not be a measurement.

- If the patient is probably asleep, and the estimation does not strongly indicate hypoglycemia, there should be no measurement.

- If the patient is about to go to sleep, there should be a measurement, unless both hypo-, and hyperglycemia is highly unlikely.

- If there is a hyperglycemic episode, and the blood glucose levels are assumed to be increasing, calibration is needed.

- If there is a hypoglycemic episode, or the patient is close to a hypoglycemic episode, calibration is needed.

- If the patient is in or close to a hyperglycemic episode, and the blood glucose levels are assumed to be increasing, calibration is needed.

- If the patient is close to a hyperglycemic episode, but the blood glucose levels are assumed to be decreasing, measurement is not needed.

- If the patient is close to a hypoglycemic episode, but the blood glucose levels are assumed to be increasing, measurement is not needed.

It is needless to see that the actual parameters of this module can depend on various factors, such as lifestyle or sensor properties. Furthermore, the parameters of the fuzzy sets should reflect the uneven significance of hypo- and hyperglycemia.

\section{Results}

Simulations were conducted to show the capabilities of the proposed system. The numerical values of the model parameters were randomly chosen from the 
6 patient parameter sets presented in [12] and 50 virtual patient parameter sets generated using the parameter bounds also presented in [12]. The meal intake and physical activity was randomized as well. Each simulation covered 48 hours and assumed unusually high carbohydrate (CHO) intake (180g $\mathrm{CHO}$ to $310 \mathrm{~g} \mathrm{CHO})$. The details are summarized in Table 1. Furthermore there is $50 \%$ chance of physical activity starting between 9:00-12:00 for 1-4 hours. Uniform distribution was used in all cases. The SGQF is assumed to have $10 \%$ initial estimation error, and that the patient does not miss any of the manual measurements requested by the so-created Calibration Supervisory unit. Fig. 1 1 shows an example for one of the simulations. The acquired results are summarized in Table 2.

Table 1. High carbohydrate intake simulation parameters

\begin{tabular}{|l|c|c|c|c|c|c|}
\cline { 2 - 7 } \multicolumn{1}{c|}{} & Breakfast & Snack 1 & Lunch & Snack 2 & Dinner & Snack 3 \\
\hline Chance of occurrence & $100 \%$ & $50 \%$ & $100 \%$ & $50 \%$ & $100 \%$ & $50 \%$ \\
\hline Amount $[g]$ & $40-60$ & $5-25$ & $70-110$ & $5-25$ & $55-75$ & $5-15$ \\
\hline Time $[$ hour $]$ & $6-10 \mathrm{am}$ & $8-11 \mathrm{am}$ & $11 \mathrm{am}-3 \mathrm{pm}$ & $3-6 \mathrm{pm}$ & $6-10 \mathrm{pm}$ & $10 \mathrm{pm}-12 \mathrm{am}$ \\
\hline
\end{tabular}

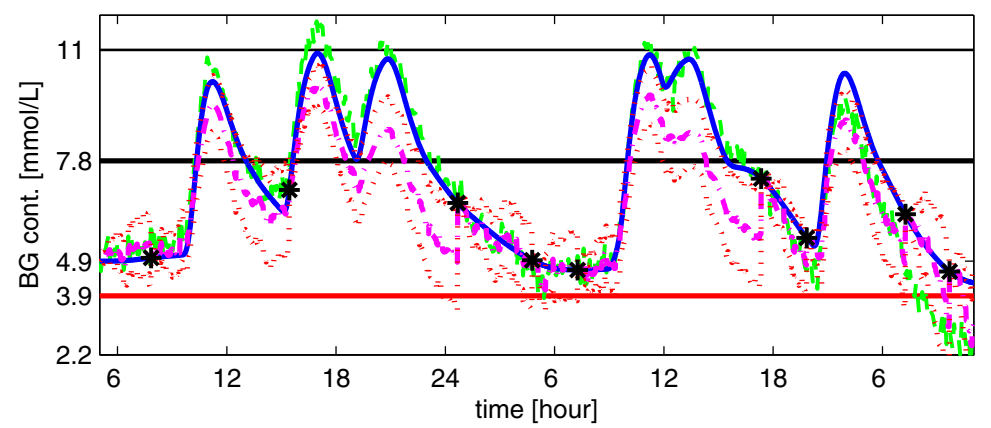

Fig. 1. Example of simulation results. The real (solid blue line), measured (dashed green line) and estimated (dash-dotted magenta line) blood glucose concentration are displayed, as well as the estimated error bounds (dotted red line) and the instances of the manual measurement (black asterisk). The three solid horizontal lines in the background mark the upper $(7.8 \mathrm{mmol} / \mathrm{L})$ and lower $(3.9 \mathrm{mmol} / \mathrm{L})$ bounds of the normoglycemic region, as well as the threshold for severe hyperglycemia $(11 \mathrm{mmol} / \mathrm{L})$.

Table 2. Simulation results for high CHO intake scenario. The percentage represents the time spent in the designated regions through all 500 Monte Carlo simulations.

\begin{tabular}{|c|c|}
\hline Hypoglycemia $(<3.9 \mathrm{mmol} / \mathrm{L})$ & $1.10 \%$ \\
\hline Normoglycemia $(3.9-6 \mathrm{mmol} / \mathrm{L})$ & $33.56 \%$ \\
\hline Mild hyperglycemia $(6-7.8 \mathrm{mmol} / \mathrm{L})$ & $26.01 \%$ \\
\hline Hyperglycemia $(6-11 \mathrm{mmol} / \mathrm{L})$ & $63.26 \%$ \\
\hline Severe hyperglycemia $(>11.1 \mathrm{mmol} / \mathrm{L})$ & $2.08 \%$ \\
\hline \hline Average number of calibrations & 10.91 \\
\hline
\end{tabular}




\section{Conclusion}

The proposed controller, aided by the sparse-grid quadrature filter and the calibration supervisor could reduce both hypoglycemic and severe hyperglycemic episodes for all virtual patients in the case of extreme meal intake and sensor drift. A more sophisticated control algorithm and the optimization of filter parameters and the calibration supervisor could improve these results further. In future works the robustness of the solution must be examined, since it is safe to assume that the used T1DM model is inaccurate, let it be parameter inaccuracy or additive/multiplicative uncertainty defined in frequency domain. Furthermore, additional safety measures must be taken if the patient is less cooperative and reliable with the sensor calibration. It is also possible to use the filter for prediction, hence providing more information for the supervisor unit.

\section{References}

1. Palerm, C.: Physiologic insulin delivery with insulin feedback: A control systems perspective. Comp. Meth. Progr. Biomed. 102, 130-137 (2011)

2. Zisser, H., Palerm, C.C., Bevier, W.C., Doyle III, F.J., Jovanovic, L.: Clinical update on optimal prandial insulin dosing using a refined run-to-run control algorithm. J. Diabetes Sci. Technol. 3, 487-491 (2009)

3. Palumbo, P., Pizzichelli, G., Panunzi, S., Pepe, P., Gaetano, A.D.: Tests on a virtual patient for an observer-based, closed-loop control of plasma glycemia. In: 50th IEEE CDC-ECC Conference, Orlando, USA, pp. 6936-6941 (2011)

4. Parker, R., Doyle, F., Ward, J., Peppas, N.: Robust $\mathcal{H}_{\infty}$ glucose control in diabetes using a physiological model. AIChE J. 46, 2537-2549 (2000)

5. Femat, R., Ruiz-Velazquez, E., Quiroz, G.: Weighting restriction for intravenous insulin delivery on T1DM patient via $\mathcal{H}_{\infty}$ control. IEEE T. Autom. Sci. Eng. 6, 239-247 (2009)

6. Hovorka, R., Canonico, V., Chassin, L., Haueter, U., Massi-Benedetti, M., Federici, M.O., Pieber, T., Schaller, H., Schaupp, L., Vering, T., Wilinska, M.: Nonlinear model predictive control of glucose concentration in subjects with type 1 diabetes. Physiol. Meas. 25, 905-920 (2004)

7. Kovatchev, B., Cobelli, C., Renard, E.: Multi-national study of subcutaneous model-predictive closed-loop control in type 1 diabetes: summary of the results. J. Diabetes Sci. Technol. 4, 1374-1381 (2010)

8. Kovács, L., Benyó, B., Bokor, J., Benyó, Z.: Induced $\mathcal{L}_{2}$-norm minimization of glucose-insulin system for type I diabetic patients. Comp. Meth. Progr. Biomed. 102, 105-118 (2011)

9. Pena, R.S., Ghersin, A., Bianchi, F.: Time-varying procedures for insulindependent diabetes mellitus control. J. Electr. Comp. Eng. 2011, 1-10 (2011)

10. Phillip, M., Battelino, T., Atlas, E., Kordonouri, O., Bratina, N., Miller, S., Biester, T., Stefanija, M., Muller, I., Nimri, R., Danne, T.: Nocturnal glucose control with an artificial pancreas at a diabetes camp. N. Engl. J. Med. 368, 824-833 (2013)

11. Zarkogianni, K., Vazeou, A., Mougiakakou, S., Prountzou, A., Nikita, K.: An insulin infusion advisory system based on autotuning nonlinear model-predictive control. IEEE J. Biomed. Eng. 58, 2467-2477 (2011) 
12. Wilinska, M., Chassin, L., Acerini, C., Allen, J., Dunger, D., Hovorka, R.: Simulation environment to evaluate closed-loop insulin delivery systems in type 1 diabetes. J. Diabetes Sci. Technol. 4, 132-144 (2010)

13. Szalay, P., Eigner, G., Kozlovszky, M., Rudas, I., Kovács, L.: The significance of LPV modeling of a widely used T1DM model. In: EMBC 35th Annual International Conference of the IEEE, Osaka, Japan, pp. 3531-3534 (2013)

14. Lantos, B.: Theory and design of control systems II. Akademia, Budapest (2003)

15. Jia, B., Xin, M., Cheng, Y.: Sparse-grid quadrature nonlinear filtering. Automatica $48,327-341$ (2012)

16. Arasaratnam, I., Haykin, S., Elliot, R.: Discrete-time nonlinear filtering algorithms using Gauss-Hermite quadrature. Proc. IEEE 95, 953-977 (2007) 